\title{
Cold dark matter heats up
}

\author{
Andrew Pontzen ${ }^{1,2,3}$, Fabio Governato ${ }^{4}$ \\ ${ }^{1}$ Department of Physics and Astronomy, University College London, London WC1E 6BT \\ ${ }^{2}$ Oxford Astrophysics, Denys Wilkinson Building, Keble Road, Oxford, OX1 3RH \\ ${ }^{3}$ Balliol College, Broad Street, Oxford, OX1 3BJ \\ ${ }^{4}$ Astronomy Department, University of Washington, Seattle, WA 98195, US
}

Draft review as submitted to Nature on 1 Oct 2013.

Accepted version scheduled for publication on $13 \mathrm{Feb} 2014$. In accordance with Nature policies, the accepted version cannot be posted until 13 Sep 2014.

One of the principal discoveries in modern cosmology is that standard model particles (including baryons, leptons and photons) together comprise only $5 \%$ of the mass-energy budget of the Universe ${ }^{1}$. The remaining $95 \%$ consists of dark energy and dark matter (DM). Consequently our picture of the universe is known as $\Lambda$ CDM, with $\Lambda$ denoting dark energy and CDM cold dark matter. $\Lambda$ CDM is being challenged by its apparent inability to explain the low density of DM measured at the centre of cosmological systems, ranging from faint dwarf galaxies to massive clusters containing tens of galaxies the size of the Milky Way. But before making conclusions one should carefully include the effect of gas and stars, which were historically seen as merely a passive component during the assembly of galaxies. We now understand that these can in fact significantly alter the DM component, through a coupling based on rapid gravitational potential fluctuations.

Despite the unknown nature of the dominant components, $\Lambda \mathrm{CDM}^{2}$ successfully describes the evolution of the Universe from its near-uniform early state, as measured by the cosmic microwave background $^{1}$, to the present-day clustered distribution of matter ${ }^{3}$ in an accelerating Universe. Consequently the properties of dark matter and the processes driving the formation and evolution of galaxies are fundamental, closely connected problems in modern astrophysics.

$\Lambda \mathrm{CDM}$, through its explanation of observations on the largest observable scales, has been established as the standard cosmological paradigm. Over time increasingly massive dark matter 'halos' form through gravitational instabilities, starting from small, linear perturbations in the matter density field. It is within the gravitational potential of DM halos that galaxy formation - gas cooling and star formation - proceeds 4 . However, long-standing problems have been encountered in reconciling the predictions of $\Lambda \mathrm{CDM}$ with observational results at galaxy scales. These problems likely stem from our poor knowledge of the complex physics associated with star formation, and are complicated by failure to identify the DM particle candidate.

The goal of the present review is to present recent progress in solving the discrepancies. We now understand that gas outflows from galaxies are ubiquitous, powered by energy released from stars and black hole accretion. These outflows change the distribution of the gas and stars which subsequently form. If the outflows launch at sufficient speed, they also cause an irreversible change in the dark matter distribution, even if the gas later returns to the galaxy in a "fountain". These processes fundamentally modify the structure of galaxies, and serve to bring theoretical expectations into agreement with previously problematic observational constraints. It is therefore important to fully understand the relevant astrophysics before using galaxies to place constraints on dark matter candidate particles.

\section{Galaxy formation with collisionless cold dark matter}

The viability of the $\Lambda \mathrm{CDM}$ picture of structure formation was first evaluated using computer simulations (allowing, for instance, neutrinos to be ruled out as the dominant component of dark matter ${ }^{5}$ ). Gas cooling and star formation within DM halos is now the standard paradigm for the origin of galaxies ${ }^{4}$. The behaviour of DM can be simulated on computers by chunking a portion of the universe into "particles" and evolving. Since the particles interact only through gravity, these simulations are called collisionless.

Early attempts used just 30000 particles to follow large regions of the Universe. Consequently one particle had the mass of a large galaxy - even so, such simulations were expensive, taking $70 \mathrm{CPU}$ hours on state-of-the-art $3 \mathrm{MHz}$ facilities. Such calculations would now take a few minutes on a cellphone. The growth of computing power and parallel capabilities meant that, by the 1990s, simulations became sufficiently powerful to make detailed predictions of the internal structure of halos in different cosmological scenarios. These simulations highlighted the universal nature of DM halos 

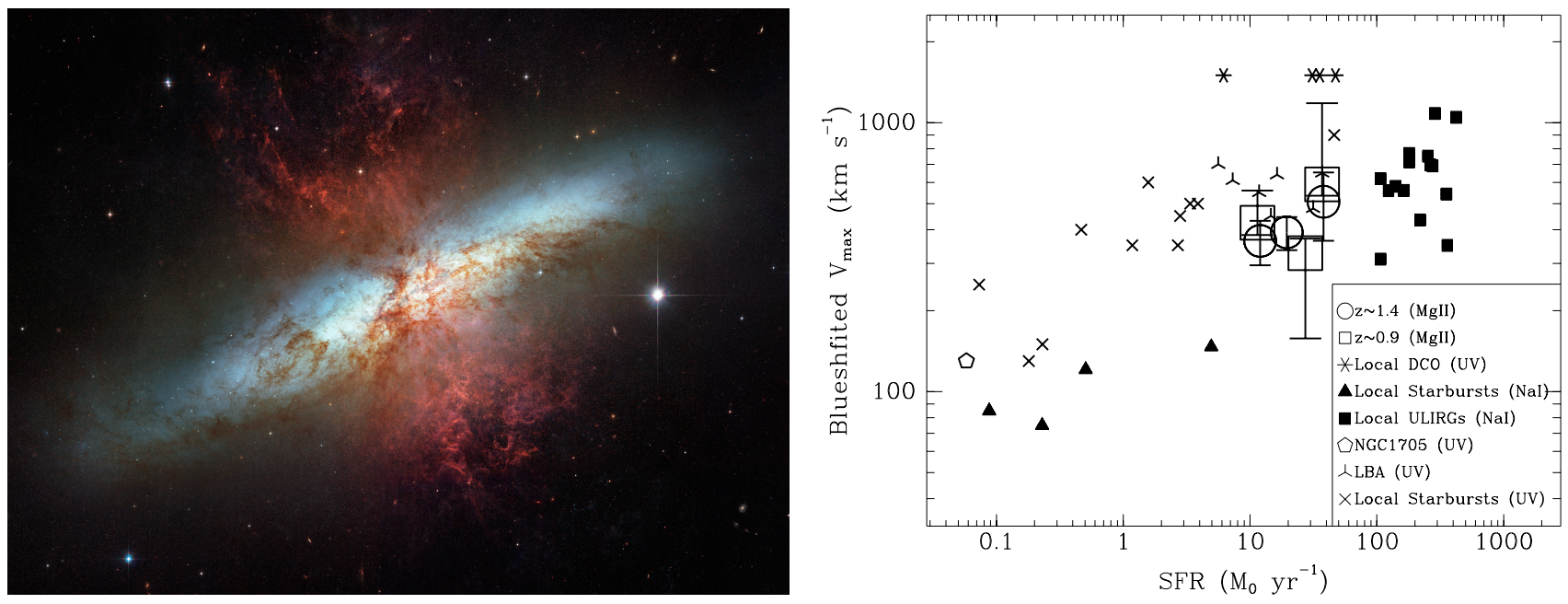

Figure 1: The left panel (by J. Gallagher) shows a composite image of M82 taken by the Hubble Space Telescope. Purple colours correspond to narrow-band $\mathrm{H} \alpha$ emission, allowing us to see recombining hydrogen in outflowing gas. The right panel, from Martin et al 29 , shows a compilation of measured absorption line blue-shifts for cool gas as a function of the galaxy's star formation rate. Even dwarf galaxies with star formation rates under $1 \mathrm{M}_{\odot} \mathrm{yr}^{-1}$ are able to support winds exceeding $100 \mathrm{~km} \mathrm{~s}^{-1}$. The outflow rate of these winds is typically several times the instantaneous star formation rate of the parent galaxy.

formed through collisionless collapse. The spherically-averaged density of halos is 'cusped' at the centre (scaling approximately as $\rho \propto r^{-1}$ ), rolling to a steeper slope at larger radius (reaching $\rho \propto r^{-3}$ ); such behaviour is known as "NFW" after the authors of a pivotal paper ${ }^{6}$.

At the same time, simulations started highlighting a number of deficiencies in the CDM scenarios. The most evident was the overabundance, by more than an order of magnitude, of small satellites $\frac{7.8}{8}$ compared to the number observed orbiting the Milky Way 9 at the time. Worse, the simulations significantly overpredicted the density of DM at the centre of galaxies $\frac{10}{\text { Increas- }}$ ingly precise observations of the rotation curves of field galaxies have confirmed this discrepancy ${ }^{11}$ (see $\S 3$ ).

Collisionless DM simulations have since reached maturity, with modern simulations using several billion resolution elements for just one Milky Way sized hald 12,13. However to make predictions which are testable against observations of the real Universe, baryon physics must be introduced. (Here we are adopting the astronomical convention of referring to baryons and leptons collectively as 'baryons'.) Because baryons dissipate energy and so collapse to smaller scales than DM, they constitute a sizeable fraction of the mass in the central regions of all but the faintest galaxies 14. Moreover observational constraints on galaxy formation ultimately come from photons, which can only be sourced by baryons. Accordingly much effort has recently been devoted to implementing gas hydrodynamics and a description of star formation within simulations 15 .

The energy released by young stellar populations and active galactic nuclei into the surrounding intergalactic medium is critical for regulating star formation 4 . Without this energy, most of the gas becomes cold and dense, rapidly collapsing to form stars, contradicting observations. Processes providing the energy to halt collapse are collectively named 'feedback' and include supernova winds, radiation from young stars, and radiation and heat from black hole accretion $19 \mid 22$. Including these effects has led to strides forward in forming realistic disk galaxies, reproducing the efficiency of star formation as a function of galaxy mass, and linking gas accretion and mergers to galaxy morphology $23 \sqrt[25]{25}$. However, until recently any direct effect of the baryonic component on the DM was limited to a minor 'adiabatic' correction ${ }^{26}$ (see box A). In other words, star formation (SF) processes resulted in 'passive' changes to the galaxy population - modulating the star formation rate without significant changes to the underlying cosmic DM scaffolding.

This picture has recently been subverted. Spectroscopic observations reveal the ubiquity of massive galaxy outflows driven by feedback, carrying significant gas mass away from star forming galaxies throughout cosmic history 27.|29 (see Section 22. It has slowly been realised that these directly observed processes have a non-adiabatic impact on the associated dark matter halos. The effect is to relieve discrepancies between baseline CDM simulations and the real Universe (discussed in Section 3). The emerging understanding of these processes constitute the central part of this review (Section 4 ).

\section{Evidence of galaxy outflows and its effect on the stellar component of galaxies}

There is clear observational evidence that star formation activity drives gas out of galaxies (Figure 11). This largely arises from studies of the resonance absorption lines imprinted into spectra by the presence of heavy elements. Consequently dramatic advances in our knowledge have been made possible by $10 \mathrm{~m}$-class telescope spectroscopy with instruments including Keck DEIMOS 28 

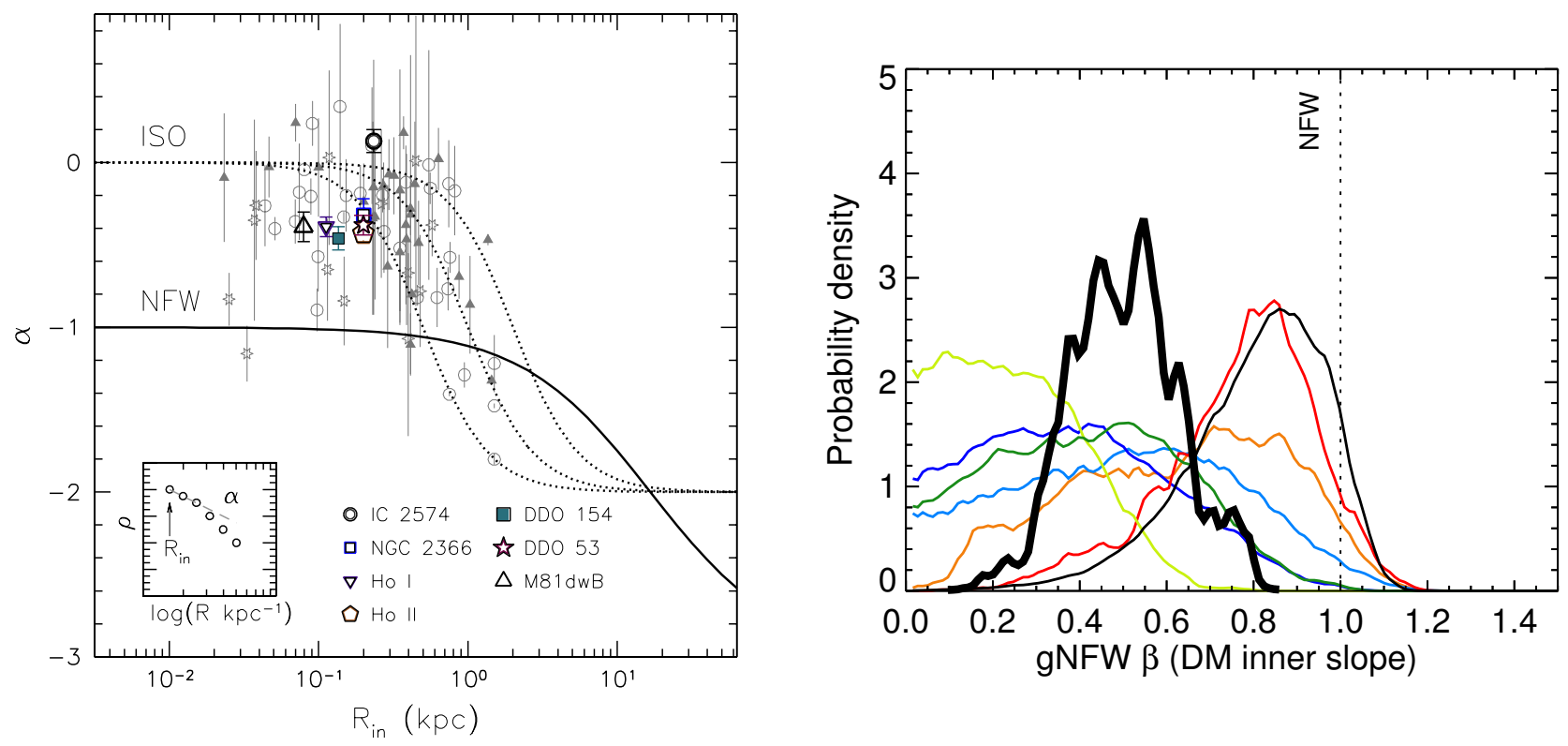

Figure 2: The left panel is a compilation $\frac{51}{1}$ of observed innermost dark matter density profile slopes $\left(\alpha\right.$ where $\left.\rho_{\text {DM }}(r) \propto r^{\alpha}\right)$ for field dwarf galaxies, plotted at the innermost point where a robust determination has been achieved. Where the slope $\alpha$ can be measured interior to around one kiloparsec, it is typically much shallower $(\alpha>-1)$ than the simulated "NFW" result. The right pane ${ }^{77}$ shows the probability distribution function on the parameter $\beta=-\alpha$ for a selection of galaxy clusters $\left(M \sim 10^{15} \mathrm{M}_{\odot}\right)$. While the constraints on individual clusters are quite broad, the combined constraints (thick line) again indicate a shallower-than-NFW slope.

and LRIS ${ }^{30}$. One can either look for blue-shifted absorption in the spectra of galaxies themselves 31 or as 'intervening' features in the spectra of background quasars 29 . A natural source for the energy required to generate these outflows is supernovac ${ }^{[32}$ and ionising radiation 33 associated with stellar populations. In addition, energy released during accretion onto a massive central black hole may have a role to play, although the available energy is thought to scale steeply with the black hole's mass, limiting these effects to the brightest galaxies or their progenitors 1920 .

Recent results underline the ubiquity of outflows 35 and show that their speed likely scales with the star formation rate of the associated galaxy (see Figure 1). Galaxies are surrounded by enriched gas moving at hundreds of kilometres per second $\frac{\sqrt{36}}{\text { in bubbles }}$ extending to $100 \mathrm{kpc}$ or more. This result is exceptionally hard to explain without significant galactic winds. Mounting evidence also suggests that much of the in-flowing material into galaxies may also be metal-enriched 37 , consistent with a picture in which much of the wind does not attain the escape velocity but instead re-accretes 38,39 .

A separate argument also points to the importance of winds during galaxy formation. Observed stellar profiles of small galaxies are mostly 'bulgeless', i.e. well approximated by a disk of gas and stars with an almost exponential profile 4041 . Yet cosmological simulations show that the dark matter and baryons accumulated in all galaxy halos contain a large fraction of low angular momentum materia ${ }^{42}$ - which would imply the presence of a bulge $e^{43}$. This problem, known as the 'angular momentum catastrophe', is solved if low angular momentum gas is ejected $\sqrt[44,45]{ }$ by winds at relatively high-z when SF peaks 4 . This makes the physics of galactic winds of fundamental importance to understanding the population of disk galaxies, even before the effect on DM is considered.

\section{Evidence for a cusp-core discrepancy}

We now turn our attention to the excessive quantity of dark matter predicted by the CDM model compared to measured densities in the innermost regions of galaxies and clusters.

Dwarf galaxies As explained above, the under-abundance of dark matter in the centre of dwarf galaxies relative to theoretical predictions is known as the cusp-core discrepancy. The problem was discovered as soon as cosmological simulations became capable of predicting halo structure 47,48 . Although acceptance was gradual, it is now firmly established that robust measurements of the dark matter density can be made from rotation curves of gas-rich dwarf galaxies 'in the field' (i.e. away from the influence of larger galaxies). In the innermost regions $r \lesssim 0.5 \mathrm{kpc}$ the baryonic contribution to the potential is comparable to that of the dark matter and must be subtracted ${ }^{1149}$. Consequently inferring the dark matter density requires (1) high spatial resolution of the gas and stellar kinematics (2) a comprehensive understanding of how to estimate and subtract the stellar and gas mass distribution from the central kiloparsec and (3) careful handling of systematic observational errors. The last category encompasses possible biases arising from radio beam-smearing, departure from circular orbits, centring difficulties, unknown details of stellar mass-to-light ratios and gravita- 
tional potential asphericity within galaxies; these are now thought to be under control, since we can test algorithms on mock observations from simulations (where the true density is known) 50 .

Results from recent surveys of the local Universe such as THINGS and LITTLE THINGS 53:54 can therefore be regarded as free from significant observational bias. These samples reveal shallower-than-NFW dark matter profiles in a large fraction of dwarf field galaxies, with $\rho \propto r^{-0.4}$ interior to $r \simeq 1 \mathrm{kpc}$ (Figure 2 left panel). The objects are referred to as 'cored' although the estimated density profile is almost never actually flat. After 20 years of study the cusp-core problem has remained a persistent and significant discrepancy between theoretical models of a $\Lambda \mathrm{CDM}$ universe and observations of dwarf galaxies.

Milky Way Satellites Small galaxies known as 'dwarf spheroidals' orbit close to the Milky Way. The dwarf spheroidals have little gas content and their stellar content is not in a rotational disk 9 . This likely reflects the effect of tidal fields and strong interactions with the hot gas in the halo of the parent galaxy 55 . Sampling the smallest halo masses in which galaxies form, these satellites have the potential to constrain the properties of dark matter and the physics of galaxy formation and have accordingly received significant attention 56 .

We discussed above how field dwarfs have been fundamental in revealing the apparent over-concentration of DM at the centre of halos. Satellite dwarfs, with an order of magnitude fewer stars still, are potentially powerful probes of the DM distribution at the smallest scales 57 . Various techniques hint at the existence of cores, rather than cusps, in the brightest dwarf spheroidals 58 . However because galaxy satellites do not possess HI disks and deviate from spherical symmetry, inferring the mass distribution of their DM halos is significantly harder than for field galaxies. Simpler is to measure total mass inside the half-light radius (which typically lies at a few hundred parsecs $\sqrt{61}$. Compared to the most massive satellites in CDM it is widely believed that there is too little mass in each real dwarf spheroidal, a problem which is referred to as the objects being "too big to fail" 62. However the effect of tidal forces and stripping 63.64 complicate the interpretation. At present the properties and abundance of isolated, small field galaxies provide stronger constraints on models of SF and feedback and alternative DM models 56 .

High mass galaxies and galaxy clusters Field dwarfs typically fall into the category of "low-surface-brightness" galaxies, defined by their extended diffuse stellar and gaseous disks. The uncertainties (discussed above) in recovering dark matter distributions in these objects are mitigated by the relatively small baryonic contribution to the potential at the time they are observed. A fraction of more massive galaxies (with rotational peak velocities larger than $100 \mathrm{~km} \mathrm{~s}^{-1}$ ) also have these characteristics. Analysis of such galaxies ${ }^{67}$ again point to relatively flat central DM profiles. This is a significant finding because it shows that cores can be formed in halos with estimated stellar masses up to $5 \times 10^{9} \mathrm{M}_{\odot}$.

The inner distribution of DM in galaxies with more conventional, massive disks (similar to our own Milky Way, for instance) is unfortunately harder to ascertain because the gravitational potential is more strongly dominated by baryons $\frac{14}{}$, so that uncertainties in the age, metallicity and hence light-to-mass conversion ratios of stellar populations dominate. However, many attempts have pointed to smaller central dark matter densities than theoretically expected 68 , in line with the low-surface-brightness results. Some observations point to well defined scaling laws that link the DM and baryon components, with DM and baryons following similar profiles 69 . The significance of this relation is still very poorly understood but it may point to a tight coupling between baryons and DM at galactic scales ${ }^{70}$. More indirect constraints on the central DM densities in luminous galaxies arise from the existence of stellar bars 71 which, over cosmological timescales, seem dynamically incompatible with the presence of cuspy dark matter halos $\frac{72}{\text {. }}$.

The largest bound systems in the Universe, galaxy clusters, have a mass $\gtrsim 10^{14} \mathrm{M}_{\odot}$, comparable to a hundred or more Milky Way galaxies. The dark matter distribution in these objects can now be measured by a number of independent techniques, making them one of the most interesting cosmic laboratories to study baryon and DM interactions. Their central density is sufficiently high that strong gravitational lensing ${ }^{73}$ constrains the mass on scales of $\sim 10$ to $100 \mathrm{kpc}$; statistical weak gravitational lensing 74 can be applied on scales between $100 \mathrm{kpc}$ and a few Mpc; and information from the kinematics of member galaxies and the brightest cluster galaxy (BCG) stars ${ }^{75}$ or gas distributior $\frac{76}{76}$ gives constraints at scales of below $\sim 10 \mathrm{kpc}$. Taken together, these multi-wavelength observations provide tracers of the total density profile over multiple scales of interest, from a few kiloparsecs outwards. Current state-of-the-art studies that combine the above approaches have recovered a total density profile that is essentially compatible with NFW at all scales; however, once the stars of the BCG are subtracted, the dark matter has a central profile shallower than $\mathrm{NFW}$ on scales of $\sim 10 \mathrm{kpc}$. A wide range of possible explanations for these indications of a 'universal profile' not for DM, but rather for collisionless matter (comprising stars and DM together) have been proposed. We will explore these in Section 4

\section{Gravitational interactions between baryons and DM}

We have outlined above the observational evidence pointing towards systematic departures of the distribution of dark matter from the original expectations of the CDM paradigm. It has been widely suggested that this discrepancy could be addressed by gravitational interactions (the only way baryons and CDM can interact) that transfer energy from the baryon component to the diffuse dark matter ${ }^{78}$. If sufficient energy can be given gravitationally to dark matter particles in the centre of the halo, they will then migrate outwards, reducing the central density (note that this process will also apply to the stellar componen ${ }^{79}$ ). Energy can be transferred between these two components in two distinct ways: from the kinetic energy of incoming material or from baryonic processes linked to feedback within the galaxy. We will tackle these possibilities in turn.

As dense clumps move through a diffuse DM background a fraction of the orbital energy of the incoming material is lost to internal energy of the diffuse halo through "dynamical friction" 82 (see Box A for an explanation). The sinking of dense gaseous or stellar 
Box A - how gas affects dark matter through gravity

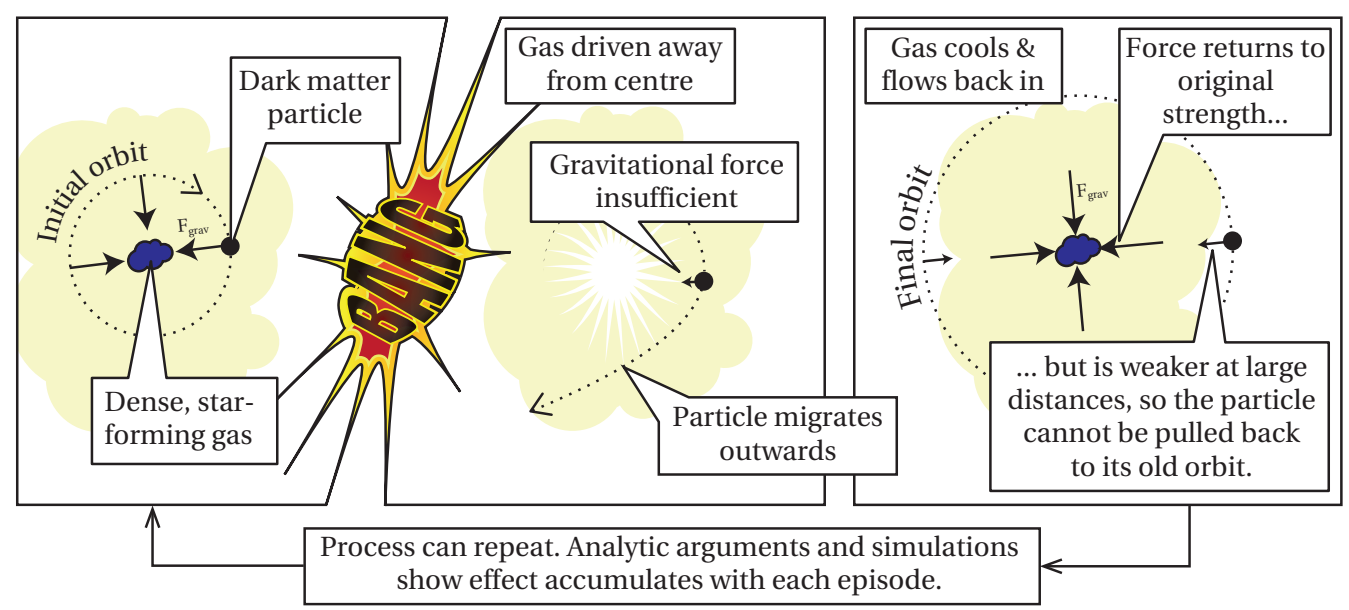

The simplest known mechanism with which baryons and DM exchange energy through gravity is called adiabatic contraction ${ }^{26}$. The word 'adiabatic' refers to a slow deepening of the gravitational potential as gas gradually accumulates in the centre of a dark matter halo on timescales longer than the local dynamical timescale. The added gravitational attraction of the accumulated material causes the dark matter to contract.

If gas arrives not in a smooth flow but in dense, discrete chunks (i.e. infalling satellite protogalaxies), this picture may be qualitatively modified by dynamical friction 83 . This effect is usually pictured as a gravitationally-induced density wake behind infalling dense clumps - the wake pulls back on the clump with the result that the kinetic energy of the clump is transferred into the dark matter.

The assumptions underlying adiabatic modelling can also fail due to outflows if these evacuate gas at speeds significantly exceeding the local circular velocity 78 . Under the adiabatic approximation, removing gas would be expected to simply reverse the effects of accumulating it in the first place, so that the final energy of any given dark matter particle would be unchanged 87 . However if the removal proceeds sufficiently quickly, net energy is transferred into the dark matter 80.89 . Moreover this transfer is irreversible in the sense that re-accreting

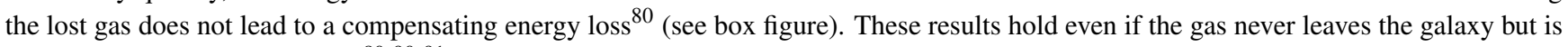
simply moved in bulk internally 80,9091 .

The reason for this is as follows 80 . Consider a dark matter particle that orbits close to the centre of the halo, where the gas is dense. If the gas is locally removed on a short timescale, the gravitational centripetal force holding the dark matter in its orbit instantaneously vanishes (or, rather, is substantially reduced in magnitude). The dark matter particle responds by flying outwards. Even if the gas later returns, the dark matter particle resides further away from the centre by the time this reversal occurs. The $1 / r^{2}$ law of gravity means the increase in force felt by the particle is quite small compared to the force originally holding the particle near the centre. The particle therefore continues to live at a large radius; this implies a net gain in energy. Repeating the process has an accumulative effect, which allows a significant transformation to be accomplished by recycling a small amount of gas instead of expelling an unfeasibly large amount of gas in one episode.

clumps can flatten the central DM profile over a range of scales, although significant core creation has only been demonstrated in simulations of galaxy clusters rather than at the scale of individual galaxies 86 . Note that dense, centrally-concentrated baryons in in-falling clumps are an essential pre-requisite in this process.

The second class of energy sources comes from within the galaxy itself: energy liberated from stellar populations can be large compared to the binding energy of the galaxies ${ }^{44}$. Early work suggested that removing most of the baryons in a rapid, dramatic starburst event could over-compensate for the previous adiabatic contraction, leading to the desired effect of reducing the central DM density ${ }^{78}$. Subsequent works studied the feasibility of this mechanism in more detai $\frac{87.89}{}$, showing in particular that repeated outflow episodes interspersed by reaccretion had a cumulative effect on the dark matter ${ }^{89}$.

However these early investigations were limited by the unknown behaviour of gas in dwarf galaxies over cosmic time, and the lack of any clear analytic framework for understanding the apparently irreversible response of the dark matter. It was unclear even to what extent the available energy in stellar populations couples to the gas through heating and radiation pressure; consequently the idea of energy transfer from baryons to the DM was not widely accepted at this stage.

Other authors 9091 showed that gas remaining fully within the system can still be effective in removing cusps when coupled to an energy source such as stellar feedback. For instance supernovae driving gas on timescales close to the local orbital period was identified as a mechanism to transfer energy to dark matter 
particles 91 . In this case the cusps were destroyed in an energetically consistent manner without requiring any unrealistically dramatic outflows. By 2008 advances in numerical resolution and understanding of how gas cools before forming stars allowed for realistic treatments of the relevant hydrodynamics (Box B). Simulations at high redshift ${ }^{92}$ showed that dark matter could indeed be expelled self-consistently from the central regions of small protogalactic objects. This work provided the first proof-of-concept in a cosmological setting, but did not make predictions of observable objects (dwarfs, for being faint, are only observable in the nearby, redshift-zero Universe).

As it became possible to resolve star forming regions ${ }^{93}$ throughout the assembly of a dwarf galaxy from the young universe to the present day, for the first time simulations formed galaxies with stellar, gas and dark matter distributions consistent with observational bounds $35 \mid 9495$. Multiple short, locally concentrated bursts of star formation were the key new phenomenon enabling modification of the DM distribution: by temporarily evacuating gas from the central kiloparsec of the galaxy these cause dark matter to migrate irreversibly outwards ${ }^{80}$, see Box A. The actual process in play thus combines characteristics of the multiple-epoch outflow picture 89 and the internal-motions picture 91 . It does not require fine-tuning of the gas velocity or dramatic evacuation of the gas from anything but the innermost region. The key requirement is that the gas exit the centre of the galaxy faster than the local circular velocity.

Analytic modelling of multiple, impulsive changes to the gravitational potential gives considerable insight into how these changes arise and why they are irreversible $e^{80}$. This allows for an accumulation of effects as the process repeats in several gas outflow events. In a single event the total gas mass in the galaxy limits the effect of outflows ${ }^{87}$ but when the same gas is recycled and used in multiple events the only practical limitation is the total energy liberated from stellar populations and black holes (see below). The model of core creation through repeated outflows draws strong support from both analytic arguments ${ }^{80}$, and simulations using different numer-

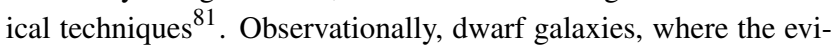
dence for cores is strongest, are observed to be gas rich and show evidence for repeated small bursts and prolonged star formation histories 96 . This supports a picture where the effect on the dark matter builds up over several Gyrs 80 , during which gas is being cycled in repeating outflow and cooling episodes.

Scaling with mass and the significance of satellite galaxies A key part of confirming which mechanisms are responsible for flattened dark matter profiles is to predict and understand in detail how the processes affect systems of differing mass. Building on the impulsive picture $\sqrt{80}$, full numerical simulations 94 and analytic argument 57 have all pointed to a transition between core creation and persistent cusps below a critical stellar mass. This dividing line likely lies between $10^{6}$ and $10^{7} \mathrm{M}_{\odot}$ (assuming most of the energy available from supernovae is transferred to the dark matter). For less massive stellar systems, the direct effects of stellar feedback on the dark matter should be minor on energetic grounds alone $e^{97}$, as SF becomes less efficient; see Figure 3 The energetic argument shows that the possible cores from supernova feedback would be indetectably small for stellar masses significantly below $10^{6} \mathrm{M}_{\odot}$.

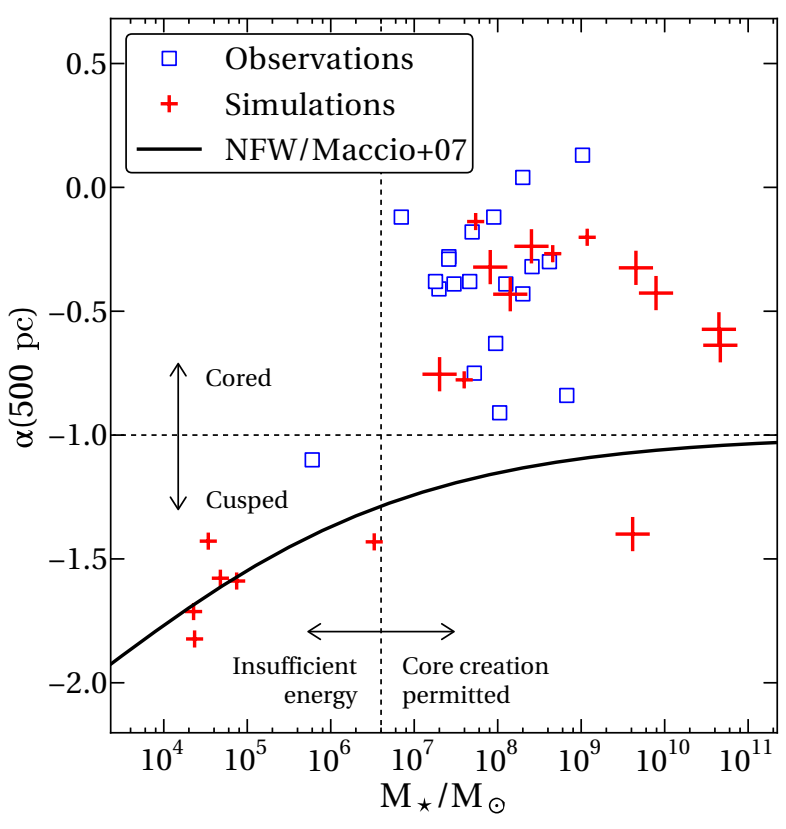

Figure 3: The log slope of the dark matter density (at radius $500 \mathrm{pc}$ ) plotted against mass of stars formed (updated from Governato et $\mathrm{a}^{\mathrm{94}}$ ). The expected slopes from pure dark matter calculations are approximated by the solid line, while hydrodynamic simulations have shallower slopes indicated by the crosses. When less than $\sim 10^{6.5} \mathrm{M}_{\odot}$ of gas has formed into stars, there is insufficient energy available to flatten the cusp ${ }^{97}$. The boxes show data from the THINGS survey 51 of field dwarf galaxies. Additional observational data at stellar masses lower than $10^{6} \mathrm{M}_{\odot}$ would be highly valuable.

For stellar masses exceeding $10^{7} \mathrm{M}_{\odot}$, it is clear that energy from SF processes is available to alter the central regions of the dark matter halo through sufficiently rapid galactic fountains or outflows ${ }^{94}$, but few simulations of luminous galaxies reach the resolution necessary to study the formation of cores. The Eris simulation (a high resolution simulation of a Milky-Way analogue) has recently been reported $\frac{98}{9}$ to have a dark matter core on scales of around $1 \mathrm{kpc}$. On the other hand it has been reported that cores shrink with respect to the halo scale radius ${ }^{99}$ for masses exceeding $10^{11} \mathrm{M}_{\odot}$ (the Milky Way mass is $\sim 10^{12} \mathrm{M}_{\odot}$ ). These statements may be reconcilable; further higher resolution work is required for progress in our understanding. As masses continue to increase to the cluster scale (see Section 3, further processes become interesting. For instance numerical work has shown that accretion onto the central black hole, if proceeding in repeated, highly energetic bursts, replicates the effect of supernovae on dwarf galaxies 100 .

The alternative: modified dark matter Many possible processes which can change the dark matter distribution in the centre of galaxies assume that the dark matter particle is cold and collisionless (i.e. interacts only through gravity) - a 'minimal' scenario. However the observational controversies detailed in Section 


\section{Box B - why high resolution gas dynamics generates outflows}

Computer simulations of the formation of galaxies would ideally resolve cosmological large scale structure (on 10's of megaparsecs) down to the scale of individual stars (at least $10^{14}$ times smaller). This is, and seems certain to remain, unfeasible.

The approach is instead to mimic the effects of stars without actually resolving them individually. Since star formation is the conclusion of run-away gas cooling and collapse, a typical computational approach is to form stars when gas satisfies certain averaged conditions, and in particular when it reaches a certain threshold density. But as resolution slowly improves in simulations, smaller regions and larger densities can be self-consistently resolved 93 .

Until the mid-2000s, a typical threshold density was set at $0.1 m_{H} \mathrm{~cm}^{-3}$, where $m_{H}$ is the mass of a hydrogen atom. This corresponds to the mean density of galactic neutral atomic gas, so stars form throughout the disc of a typical simulated galaxy. Energy output from stars in the diffuse medium results in a gentle heating of the entire galaxy, slowing the process of further star formation.

However if one can achieve sufficient resolution (and implement the more complicated cooling physics required ${ }^{15} 38,111$ ) to push to 10 or $100 m_{H} \mathrm{~cm}^{-3}$ qualitatively different behaviour results. This is the density that corresponds to molecular clouds in our galaxy, known to be the sites where clusters of stars form. Instead of forming stars in a diffuse way through the entire disc, one now efficiently forms stars in small, isolated regions ${ }^{22 \mid 45}$, which is considerably more realistic.

When energy from the resulting stellar populations is dumped into the gas, it heats to much higher temperature than diffuse star formation achieves. It is likely that intense radiation pressure is also a significant factor ${ }^{33}$. In any case, the gas is over-pressurised by a factor of at least $\sim 100$ compared to its surroundings and expands rapidly. The combination of high initial density and explosive decompression is suitable for launching galactic-scale outflows; but it is also what allows an efficient coupling of the available energy to dark matter (box A).

3 have prompted considerable interest in non-minimal DM models. By changing the properties of the dark matter candidate particle, the predictions for the distribution within halos is altered; potentially, therefore, galaxies and galaxy clusters become an important probe of particle physics 101 . For instance, the class of warm dark matter ${ }^{102}$ models (WDM) invoke a candidate particle with non negligible residual streaming motions after decoupling (such as a sterile neutrino), suppressing the formation of small scale structure $\sqrt{103}$ and delaying the collapse of dwarf sized halos and their associated star formation to slightly later epochs 104 . On the other hand these models do not produce cores on observationally relevant scales $\sqrt{67}$ and are currently strongly constrained by the clustering of the neutral gas in the cosmic web $\frac{105}{}$. Self-interacting


scenarios with significant 'dark sector' interactions. SIDM behaves more like a collisional fluid, preventing the central high- density cusp from forming and makes the central regions more spherica 107 . Unlike in the WDM case, the number density of DM halos remains relatively unchanged even at the smallest scales 108 . The diversity of theoretical models, however, gives significant freedom in the choice of the cross section and its possible dependence on particle velocity 109 . This makes it difficult to establish a single baseline SIDM scenario.

Overall it seems that neither WDM nor SIDM on their own provide a complete alleviation of the tensions detailed in Section 3 In particular, because the infall pattern of matter is driven by the large structure, no DM model can alone alleviate the problem of removing low angular momentum baryons from the centre of galaxies without unfeasible modifications to the large scale power spectrum of matter fluctuations. But the effects of baryons may amplify or change the signatures of these particle models (or, worse, make them more similar to the prediction of the CDM model). The dwarf spheroidals teach us that different transformative mechanisms interact in surprising, non-linear ways $\sqrt{63}$, motivating a more detailed study of the galaxies formed in fully hydrodynamical simulations with WDM or SIDM.

Ideally to alleviate degeneracies between particle-physics and outflow-induced modifications to CDM, one would identify regimes in which only one or the other is active. This points towards the future value of careful studies probing scalings of cores from stellar masses below $10^{7} \mathrm{M}_{\odot}$ (where the energy available to create cores is so limited that baryonic effects are tightly constrained) to above $10^{13} \mathrm{M}_{\odot}$ (where a variety of processes are feasible).

\section{Conclusions}

The $\Lambda \mathrm{CDM}$ cosmology underlies a highly successful paradigm for explaining the formation of visible structure in the universe. Until recently, the key ingredients were passive processes which controlled the association of observable matter with the dark matter (for instance suppressing over-efficient star formation) while having little explicit effect on the underlying dark matter. There is, however, a new, rich literature of processes which violate this basic assumption and lead to fundamental modifications to the observable properties of galaxies. In the last few years these have come into sharp focus as increasingly sophisticated computer simulations have begun to follow the effects of star formation, and many relevant observational techniques have matured to the point that they can be regarded as robust. Direct evidence of precisely which 'baryonic processes' are in play and their relative importance in the real Universe at different scales should be our next priority. Because these baryonic processes simultaneously modify a number of observational diagnostics (outflows, dark matter cores, stellar morphology and star formation regulation), they weave into a coherent, testable framework.

It remains a possibility that tensions between observation and theory at the scale of faint dwarfs and clusters may point to exotic particle physics. Ultimately we expect that a concerted effort from theorists and observers can achieve the goal of pointing to unique predictions of non-minimal DM models. Of particular interest in 
the coming years will be (i) improved understanding of the dark matter in dwarf spheroidals and faint field galaxies; if cores persist at the faintest end, it is a generic conclusion that baryonic physics cannot account for them 9497; (ii) study of the stellar population ages and, separately, metallicity distributions of these objects to determine as far as possible whether the required bursty star formation histories are consistent propositions 96 ; (iii) better predictions of the scalings of cores in massive galaxies and clusters for different scenarios; (iv) observations that constrain the star formation histories of dwarfs 110 and the behaviour of gas at high redshift, especially through absorption line studies which are sensitive to internal kinematics and outflows 105 ; $(v)$ renewed effort to understand how non-minimal dark matter scenarios (such as WDM or SIDM) interact with the revised, more complex baryonic physics of galaxy formation.

Acknowledgements We would like to thank Se-Heon Oh, Simon White, Max Pettini, Crystal Martin, Matt Walker, Jorge Peñarrubia, Alyson Brooks, Tommaso Treu, Richard Ellis, James Wadsley and Lisa Randall for helpful discussions and comments on an early draft.

\section{References}

1. Planck Collaboration et al. Planck 2013 results. XVI. Cosmological parameters. A\&A submitted (2013). arXiv:1303.5076

2. Blumenthal, G. R., Faber, S. M., Primack, J. R. \& Rees, M. J. Formation of galaxies and large-scale structure with cold dark matter. Nature 311, 517-525 (1984).

3. Percival, W. J. et al. The $2 \mathrm{dF}$ Galaxy Redshift Survey: the power spectrum and the matter content of the Universe. MNRAS 327, 1297 1306 (2001). arXiv:astro-ph/ 0105252

4. White, S. D. M. \& Rees, M. J. Core condensation in heavy halos - A two-stage theory for galaxy formation and clustering. MNRAS 183, 341-358 (1978).

5. White, S. D. M., Frenk, C. S. \& Davis, M. Clustering in a neutrinodominated universe. ApJ 274, L1-L5 (1983).

6. Navarro, J. F., Frenk, C. S. \& White, S. D. M. The Structure of Cold Dark Matter Halos. ApJ 462, 563-+ (1996).

7. Moore, B. et al. Dark Matter Substructure within Galactic Halos. ApJ 524, L19-L22 (1999).

8. Klypin, A., Kravtsov, A. V., Valenzuela, O. \& Prada, F. Where Are the Missing Galactic Satellites? ApJ 522, 82-92 (1999).

9. Mateo, M. L. Dwarf Galaxies of the Local Group. Ann. Rev. Astron. Astrophys. 36, 435-506 (1998).

10. Moore, B., Governato, F., Quinn, T., Stadel, J. \& Lake, G. Resolving the Structure of Cold Dark Matter Halos. ApJ 499, L5+ (1998).

11. de Blok, W. J. G. et al. High-Resolution Rotation Curves and Galaxy Mass Models from THINGS. AJ 136, 2648-2719 (2008). 0810. 2100

12. Springel, V. et al. The Aquarius Project: the subhaloes of galactic haloes. MNRAS 391, 1685-1711 (2008). 0809.0898

13. Stadel, J. et al. Quantifying the heart of darkness with GHALO a multibillion particle simulation of a galactic halo. MNRAS 398, L21-L25 (2009). 0808 .2981

14. Bell, E. F. \& de Jong, R. S. Stellar Mass-to-Light Ratios and the Tully-Fisher Relation. ApJ 550, 212-229 (2001).
15. Gnedin, N. Y., Tassis, K. \& Kravtsov, A. V. Modeling Molecular Hydrogen and Star Formation in Cosmological Simulations. ApJ 697, 55-67 (2009). 0810.4148

16. Wise, J. H. \& Abel, T. ENZO+MORAY: radiation hydrodynamics adaptive mesh refinement simulations with adaptive ray tracing. MNRAS 414, 3458-3491 (2011). 1012 .2865

17. Teyssier, R. Cosmological hydrodynamics with adaptive mesh refinement. A new high resolution code called RAMSES. A\&A 385, 337-364 (2002). arXiv:astro-ph/0111367

18. Kereš, D., Vogelsberger, M., Sijacki, D., Springel, V. \& Hernquist, L. Moving-mesh cosmology: characteristics of galaxies and haloes. MNRAS 425, 2027-2048 (2012). 1109.4638

19. Croton, D. J. et al. The many lives of active galactic nuclei: cooling flows, black holes and the luminosities and colours of galaxies. MNRAS 365, 11-28 (2006). arXiv: astro-ph/0508046

20. Bower, R. G. et al. Breaking the hierarchy of galaxy formation. MNRAS 370, 645-655 (2006). arXiv: astro-ph/ 0511338

21. Stinson, G. et al. Star formation and feedback in smoothed particle hydrodynamic simulations - I. Isolated galaxies. MNRAS 373, 1074 1090 (2006). arXiv: astro-ph/ 0602350

22. Hopkins, P. F., Quataert, E. \& Murray, N. Stellar feedback in galaxies and the origin of galaxy-scale winds. MNRAS 421, 3522-3537 (2012). 1110.4638

23. Robertson, B. et al. A Merger-driven Scenario for Cosmological Disk Galaxy Formation. ApJ 645, 986-1000 (2006). arXiv: astro-ph/0503369

24. Kereš, D., Katz, N., Weinberg, D. H. \& Davé, R. How do galaxies get their gas? MNRAS 363, 2-28 (2005). ast ro-ph/ 0407095

25. Dekel, A. et al. Cold streams in early massive hot haloes as the main mode of galaxy formation. Nature 457, 451-454 (2009). 0808 . 0553

26. Blumenthal, G. R., Faber, S. M., Flores, R. \& Primack, J. R. Contraction of dark matter galactic halos due to baryonic infall. ApJ 301, 27-34 (1986).

27. Shapley, A. E., Steidel, C. C., Pettini, M. \& Adelberger, K. L. RestFrame Ultraviolet Spectra of z 3 Lyman Break Galaxies. ApJ 588, 65-89 (2003). arXiv: astro-ph/0301230

28. Weiner, B. J. et al. Ubiquitous Outflows in DEEP2 Spectra of StarForming Galaxies at $\mathrm{z}=1.4$. ApJ 692, 187-211 (2009). 0804 . 4686

29. Martin, C. L. et al. Demographics and Physical Properties of Gas Out/Inflows at 0.4. MNRAS, submitted (2012). 1206.5552

30. Steidel, C. C. et al. The Structure and Kinematics of the Circumgalactic Medium from Far-ultraviolet Spectra of $z^{\sim}=2-3$ Galaxies. ApJ 717, 289-322 (2010). 1003.0679

31. Heckman, T. M., Lehnert, M. D., Strickland, D. K. \& Armus, L. Absorption-Line Probes of Gas and Dust in Galactic Superwinds. ApJS 129, 493-516 (2000). arXiv: astro-ph/ 0002526

32. Mac Low, M. \& Ferrara, A. Starburst-driven Mass Loss from Dwarf Galaxies: Efficiency and Metal Ejection. ApJ 513, 142-155 (1999).

33. Murray, N., Quataert, E. \& Thompson, T. A. The Disruption of Giant Molecular Clouds by Radiation Pressure \& the Efficiency of Star Formation in Galaxies. ApJ 709, 191-209 (2010). 0906.5358

34. Wise, J. H., Abel, T., Turk, M. J., Norman, M. L. \& Smith, B. D. The birth of a galaxy - II. The role of radiation pressure. MNRAS 427, 311-326 (2012). 1206.1043

35. van der Wel, A. et al. Extreme Emission-line Galaxies in CANDELS: Broadband-selected, Starbursting Dwarf Galaxies at z $; 1$. ApJ 742, 111 (2011). 1107.5256 
36. Rubin, K. H. R. et al. The Persistence of Cool Galactic Winds in High Stellar Mass Galaxies between z 1.4 and $\sim 1$. ApJ 719, 1503-1525 (2010). 0912.2343

37. Rubin, K. H. R., Prochaska, J. X., Koo, D. C. \& Phillips, A. C. The Direct Detection of Cool, Metal-enriched Gas Accretion onto Galaxies at $\mathrm{z}^{\sim} 0.5$. ApJ 747, L26 (2012). 1110.0837

38. Shen, S., Wadsley, J. \& Stinson, G. The enrichment of the intergalactic medium with adiabatic feedback - I. Metal cooling and metal diffusion. MNRAS 407, 1581-1596 (2010). 0910.5956

39. Davé, R., Oppenheimer, B. D. \& Finlator, K. Galaxy evolution in cosmological simulations with outflows - I. Stellar masses and star formation rates. MNRAS 415, 11-31 (2011). 1103.3528

40. Dutton, A. A. On the origin of exponential galaxy discs. MNRAS 396, 121-140 (2009). 0810.5164

41. Kormendy, J., Drory, N., Bender, R. \& Cornell, M. E. Bulgeless Giant Galaxies Challenge Our Picture of Galaxy Formation by Hierarchical Clustering. ApJ 723, 54-80 (2010). 1009.3015

42. Barnes, J. \& Efstathiou, G. Angular momentum from tidal torques. ApJ 319, 575-600 (1987).

43. van den Bosch, F. C., Burkert, A. \& Swaters, R. A. The angular momentum content of dwarf galaxies: new challenges for the theory of galaxy formation. MNRAS 326, 1205-1215 (2001). arXiv: astro-ph/0105082

44. Binney, J., Gerhard, O. \& Silk, J. The dark matter problem in disc galaxies. MNRAS 321, 471-474 (2001).

45. Governato, F. et al. Bulgeless dwarf galaxies and dark matter cores from supernova-driven outflows. Nature 463, 203-206 (2010).

46. Brook, C. B. et al. Hierarchical formation of bulgeless galaxies: why outflows have low angular momentum. MNRAS 415, 1051-1060 (2011). 1010.1004

47. Flores, R. A. \& Primack, J. R. Observational and theoretical constraints on singular dark matter halos. ApJ 427, L1-L4 (1994). arXiv:astro-ph/9402004

48. Moore, B. Evidence against dissipation-less dark matter from observations of galaxy haloes. Nature 370, 629-631 (1994).

49. Simon, J. D., Bolatto, A. D., Leroy, A., Blitz, L. \& Gates, E. L. High-Resolution Measurements of the Halos of Four Dark MatterDominated Galaxies: Deviations from a Universal Density Profile. ApJ 621, 757-776 (2005). arXiv: astro-ph/ 0412035

50. Swaters, R. A., Madore, B. F., van den Bosch, F. C. \& Balcells, M. The Central Mass Distribution in Dwarf and Low Surface Brightness Galaxies. ApJ 583, 732-751 (2003). arXiv:astro-ph/ 0210152

51. Oh, S.-H., de Blok, W. J. G., Brinks, E., Walter, F. \& Kennicutt, R. C., Jr. Dark and Luminous Matter in THINGS Dwarf Galaxies. AJ 141, 193 (2011). 1011.0899

52. Oh, S.-H. et al. The Central Slope of Dark Matter Cores in Dwarf Galaxies: Simulations versus THINGS. AJ 142, 24 (2011). 1011. 2777

53. Walter, F. et al. THINGS: The H I Nearby Galaxy Survey. AJ 136, 2563-2647 (2008). 0810.2125

54. Hunter, D. A. et al. Little Things. AJ 144, 134 (2012). 1208.5834

55. Mayer, L. et al. The Metamorphosis of Tidally Stirred Dwarf Galaxies. ApJ 559, 754-784 (2001). arXiv:astro-ph/0103430

56. Nierenberg, A. M. et al. Luminous Satellites. II. Spatial Distribution, Luminosity Function, and Cosmic Evolution. ApJ 752, 99 (2012). 1202.2125
57. Strigari, L. E. et al. A common mass scale for satellite galaxies of the Milky Way. Nature 454, 1096-1097 (2008). 0808 . 3772

58. Goerdt, T., Moore, B., Read, J. I., Stadel, J. \& Zemp, M. Does the Fornax dwarf spheroidal have a central cusp or core? MNRAS 368, 1073-1077 (2006). arXiv:astro-ph/0601404

59. Walker, M. G. \& Peñarrubia, J. A Method for Measuring (Slopes of) the Mass Profiles of Dwarf Spheroidal Galaxies. ApJ 742, 20 (2011). 1108.2404

60. Breddels, M. A. \& Helmi, A. Model comparison of the dark matter profiles of Fornax, Sculptor, Carina and Sextans. A\&A, submitted (2013). 1304.2976

61. Wolf, J. et al. Accurate masses for dispersion-supported galaxies. MNRAS 406, 1220-1237 (2010). 0908.2995

62. Boylan-Kolchin, M., Bullock, J. S. \& Kaplinghat, M. The Milky Way's bright satellites as an apparent failure of LCDM. MNRAS 422, 1203 (2012). 1111.2048

63. Zolotov, A. et al. Baryons Matter: Why Luminous Satellite Galaxies Have Reduced Central Masses. ApJ 761, 71 (2012). 1207.0007

64. Arraki, K. S., Klypin, A., More, S. \& Trujillo-Gomez, S. Effects of baryon removal on the structure of dwarf spheroidal galaxies. MNRAS 438, 1466 (2014). 1212.6651

65. Papastergis, E., Martin, A. M., Giovanelli, R. \& Haynes, M. P. The Velocity Width Function of Galaxies from the 40\% ALFALFA Survey: Shedding Light on the Cold Dark Matter Overabundance Problem. ApJ 739, 38 (2011). 1106.0710

66. Ferrero, I., Abadi, M. G., Navarro, J. F., Sales, L. V. \& Gurovich, S. The dark matter haloes of dwarf galaxies: a challenge for the $\Lambda$ cold dark matter paradigm? MNRAS 425, 2817-2823 (2012). 1111.6609

67. Kuzio de Naray, R. \& Spekkens, K. Do Baryons Alter the Halos of Low Surface Brightness Galaxies? ApJ 741, L29 (2011). 1109. 1288

68. McGaugh, S. S., de Blok, W. J. G., Schombert, J. M., Kuzio de Naray, R. \& Kim, J. H. The Rotation Velocity Attributable to Dark Matter at Intermediate Radii in Disk Galaxies. ApJ 659, 149-161 (2007). arXiv:astro-ph/0612410

69. Gentile, G., Famaey, B., Zhao, H. \& Salucci, P. Universality of galactic surface densities within one dark halo scale-length. Nature 461, 627-628 (2009). 0909.5203

70. Swaters, R. A., Sancisi, R., van Albada, T. S. \& van der Hulst, J. M. Are Dwarf Galaxies Dominated by Dark Matter? ApJ 729, 118 (2011). 1101.3120

71. Pérez, I., Aguerri, J. A. L. \& Méndez-Abreu, J. Bar pattern speed evolution over the last 7 Gyr. A\&A 540, A103 (2012). 1202.1929

72. Debattista, V. P. \& Sellwood, J. A. Dynamical Friction and the Distribution of Dark Matter in Barred Galaxies. ApJ 493, L5 (1998). arXiv:astro-ph/9710039

73. Miralda-Escude, J. Gravitational lensing by a cluster of galaxies and the central cD galaxy: Measuring the mass profile. ApJ 438, 514-526 (1995).

74. Umetsu, K. et al. A Precise Cluster Mass Profile Averaged from the Highest-quality Lensing Data. ApJ 738, 41 (2011). 1105.0444

75. Łokas, E. L., Wojtak, R., Gottlöber, S., Mamon, G. A. \& Prada, F. Mass distribution in nearby Abell clusters. MNRAS 367, 1463-1472 (2006). arXiv:astro-ph/0511723

76. Allen, S. W., Schmidt, R. W. \& Fabian, A. C. The X-ray virial relations for relaxed lensing clusters observed with Chandra. MNRAS 328, L37-L41 (2001). arXiv: astro-ph/0110610 
77. Newman, A. B., Treu, T., Ellis, R. S. \& Sand, D. J. The Density Profiles of Massive, Relaxed Galaxy Clusters. II. Separating Luminous and Dark Matter in Cluster Cores. ApJ 765, 25 (2013). 1209 . 1392

78. Navarro, J. F., Eke, V. R. \& Frenk, C. S. The cores of dwarf galaxy haloes. MNRAS 283, L72-L78 (1996). arXiv:astro-ph/ 9610187

79. Read, J. I. \& Gilmore, G. Mass loss from dwarf spheroidal galaxies: the origins of shallow dark matter cores and exponential surface brightness profiles. MNRAS 356, 107-124 (2005). arXiv: astro-ph/0409565

80. Pontzen, A. \& Governato, F. How supernova feedback turns dark matter cusps into cores. MNRAS 421, 3464-3471 (2012). 1106. 0499

81. Teyssier, R., Pontzen, A., Dubois, Y. \& Read, J. I. Cusp-core transformations in dwarf galaxies: observational predictions. MNRAS 429, 3068-3078 (2013). 1206.4895

82. White, S. D. M. Dynamical friction in spherical clusters. MNRAS 174, 19-28 (1976)

83. El-Zant, A., Shlosman, I. \& Hoffman, Y. Dark Halos: The Flattening of the Density Cusp by Dynamical Friction. ApJ 560, 636-643 (2001). arXiv:astro-ph/0103386

84. Tonini, C., Lapi, A. \& Salucci, P. Angular Momentum Transfer in Dark Matter Halos: Erasing the Cusp. ApJ 649, 591-598 (2006). arXiv:astro-ph/0603051

85. Romano-Díaz, E., Shlosman, I., Hoffman, Y. \& Heller, C. Erasing Dark Matter Cusps in Cosmological Galactic Halos with Baryons. ApJ 685, L105-L108 (2008). 0808 . 0195

86. El-Zant, A. A., Hoffman, Y., Primack, J., Combes, F. \& Shlosman, I. Flat-cored Dark Matter in Cuspy Clusters of Galaxies. ApJ 607, L75-L78 (2004). arXiv:astro-ph/0309412

87. Gnedin, O. Y. \& Zhao, H. Maximum feedback and dark matter profiles of dwarf galaxies. MNRAS 333, 299-306 (2002). arXiv: astro-ph/0108108

88. Mo, H. J. \& Mao, S. Galaxy formation in pre-processed dark haloes. MNRAS 353, 829-840 (2004). arXiv: astro-ph/ 0311459

89. Read, J. I. \& Gilmore, G. Mass loss from dwarf spheroidal galaxies: the origins of shallow dark matter cores and exponential surface brightness profiles. MNRAS 356, 107-124 (2005). arXiv: astro-ph/0409565

90. Weinberg, M. D. \& Katz, N. Bar-driven Dark Halo Evolution: A Resolution of the Cusp-Core Controversy. ApJ 580, 627-633 (2002).

91. Mashchenko, S., Couchman, H. M. P. \& Wadsley, J. The removal of cusps from galaxy centres by stellar feedback in the early Universe. Nature 442, 539-542 (2006). arXiv: astro-ph/ 0605672

92. Mashchenko, S., Wadsley, J. \& Couchman, H. M. P. Stellar Feedback in Dwarf Galaxy Formation. Science 319, 174- (2008). 0711. 4803

93. Saitoh, T. R. et al. Toward First-Principle Simulations of Galaxy Formation: I. How Should We Choose Star-Formation Criteria in HighResolution Simulations of Disk Galaxies? PASJ 60, 667- (2008). 0802.0961

94. Governato, F. et al. Cuspy no more: how outflows affect the central dark matter and baryon distribution in $\Lambda$ cold dark matter galaxies. MNRAS 422, 1231-1240 (2012). 1202 . 0554

95. Munshi, F. et al. Reproducing the Stellar Mass/Halo Mass Relation in Simulated LCDM Galaxies: Theory vs Observational Estimates. ApJ 766, 56 (2012). 1209.1389
96. McQuinn, K. B. W. et al. The Nature of Starbursts. I. The Star Formation Histories of Eighteen Nearby Starburst Dwarf Galaxies. ApJ 721, 297-317 (2010). 1008 .1589

97. Peñarrubia, J., Pontzen, A., Walker, M. G. \& Koposov, S. E. The Coupling between the Core/Cusp and Missing Satellite Problems. ApJ 759, L42 (2012). 1207 . 2772

98. Kuhlen, M., Guedes, J., Pillepich, A., Madau, P. \& Mayer, L. An Off-center Density Peak in the Milky Way's Dark Matter Halo? ApJ 765, 10 (2013). 1208.4844

99. Di Cintio, A. et al. The dependence of dark matter profiles on the stellar to halo mass ratio: a prediction for cusps vs cores. MNRAS, 437, 415 (2014). 1306.0898

100. Martizzi, D., Teyssier, R., Moore, B. \& Wentz, T. The effects of baryon physics, black holes and active galactic nucleus feedback on the mass distribution in clusters of galaxies. MNRAS 422, 30813091 (2012). 1112.2752

101. Markevitch, M. et al. Direct Constraints on the Dark Matter SelfInteraction Cross Section from the Merging Galaxy Cluster 1E 0657 56. ApJ 606, 819-824 (2004). arXiv: astro-ph/0309303

102. Boyarsky, A., Lesgourgues, J., Ruchayskiy, O. \& Viel, M. Realistic Sterile Neutrino Dark Matter with KeV Mass does not Contradict Cosmological Bounds. Physical Review Letters 102, 201304 (2009). 0812.3256

103. Dalcanton, J. J. \& Hogan, C. J. Halo Cores and Phase-Space Densities: Observational Constraints on Dark Matter Physics and Structure Formation. ApJ 561, 35-45 (2001). arXiv:astro-ph/ 0004381

104. Menci, N., Fiore, F. \& Lamastra, A. Galaxy formation in warm dark matter cosmology. MNRAS 421, 2384-2394 (2012). 1201.1617

105. Viel, M., Becker, G. D., Bolton, J. S. \& Haehnelt, M. G. Warm dark matter as a solution to the small scale crisis: New constraints from high redshift Lyman- $\alpha$ forest data. Phys. Rev. D 88, 043502 (2013). 1306.2314

106. Spergel, D. N. \& Steinhardt, P. J. Observational Evidence for SelfInteracting Cold Dark Matter. Physical Review Letters 84, 37603763 (2000). arXiv:astro-ph/9909386

107. Peter, A. H. G., Rocha, M., Bullock, J. S. \& Kaplinghat, M. Cosmological Simulations with Self-Interacting Dark Matter II: Halo Shapes vs. Observations. MNRAS 430, 105 (2012). 1208 . 3026

108. Zavala, J., Vogelsberger, M. \& Walker, M. G. Constraining selfinteracting dark matter with the Milky Way's dwarf spheroidals. MNRAS L52 (2013). 1211.6426

109. Tulin, S., Yu, H.-B. \& Zurek, K. M. Resonant Dark Forces and Small Scale Structure. Phys. Rev. Lett., 110, 111301 (2013). 1210.0900

110. Pacucci, F., Mesinger, A. \& Haiman, Z. Focusing on Warm Dark Matter with Lensed High-redshift Galaxies. MNRAS 435, L53 (2013). 1306.0009

111. Christensen, C. et al. Implementing molecular hydrogen in hydrodynamic simulations of galaxy formation. MNRAS 425, 3058-3076 (2012). 1205.5567 